ARTICLE

Received 17 Feb 2016 | Accepted 7 Oct 2016 | Published 5 Dec 2016

DOI: $10.1038 /$ ncomms13477

OPEN

\title{
Interatomic Coulombic decay cascades in multiply excited neon clusters
}

K. Nagaya ${ }^{1,2}$, D. lablonskyi ${ }^{3}$, N.V. Golubev ${ }^{4}$, K. Matsunami ${ }^{1}$, H. Fukuzawa ${ }^{2,3}$, K. Motomura ${ }^{2,3}$, T. Nishiyama ${ }^{1}$ T. Sakai ${ }^{1}$, T. Tachibana ${ }^{3}$, S. Mondal ${ }^{3}$, S. Wada ${ }^{2,5}$, K.C. Prince ${ }^{6}$, C. Callegari $^{6}$, C. Miron $^{7,8}$, N. Saito ${ }^{2,9}$, M. Yabashi $^{2}$, Ph. V. Demekhin ${ }^{10}$, L.S. Cederbaum ${ }^{4}$, A.I. Kuleff ${ }^{4}$, M. Yao ${ }^{1, \ddagger} \&$ K. Ueda ${ }^{2,3}$

In high-intensity laser light, matter can be ionized by direct multiphoton absorption even at photon energies below the ionization threshold. However on tuning the laser to the lowest resonant transition, the system becomes multiply excited, and more efficient, indirect ionization pathways become operative. These mechanisms are known as interatomic Coulombic decay (ICD), where one of the species de-excites to its ground state, transferring its energy to ionize another excited species. Here we show that on tuning to a higher resonant transition, a previously unknown type of interatomic Coulombic decay, intra-Rydberg ICD occurs. In it, de-excitation of an atom to a close-lying Rydberg state leads to electron emission from another neighbouring Rydberg atom. Moreover, systems multiply excited to higher Rydberg states will decay by a cascade of such processes, producing even more ions. The intra-Rydberg ICD and cascades are expected to be ubiquitous in weakly-bound systems exposed to high-intensity resonant radiation.

\footnotetext{
${ }^{1}$ Department of Physics, Graduate School of Science, Kyoto University, 606-8502 Kyoto, Japan. ${ }^{2}$ RIKEN SPring-8 Center, 679-5148 Hyogo, Japan. ${ }^{3}$ Institute of Multidisciplinary Research for Advanced Materials, Tohoku University, 980-8577 Sendai, Japan. ${ }^{4}$ Theoretische Chemie, Physikalisch-Chemisches Institut, Universität Heidelberg, D-69120 Heidelberg, Germany. ${ }^{5}$ Department of Physical Science, Hiroshima University, 739-8526 Higashi-Hiroshima, Japan.

${ }^{6}$ Elettra-Sincrotrone Trieste, Basovizza, Trieste I-34149, Italy. ${ }^{7}$ Synchrotron SOLEIL, L'Orme des Merisiers, Saint-Aubin, BP 48, FR-91192 Gif-sur-Yvette Cedex, France. ${ }^{8}$ Extreme Light Infrastructure-Nuclear Physics (ELI-NP), 'Horia Hulubei' National Institute for Physics and Nuclear Engineering, RO-077125 Măgurele, Jud. IIfov, Romania. ${ }^{9}$ National Metrology Institute of Japan, AIST, 305-8568 Tsukuba, Japan. ${ }^{10}$ Theoretische Atom- und Molekülphysik, Institut für Physik und CINSaT, Universität Kassel, D-34132 Kassel, Germany. ${ }^{\ddagger}$ Deceased. Correspondence and requests for materials should be addressed to K.U. (email: ueda@tagen.tohoku.ac.jp).
} 
W hen an inner-valence ionized atom or molecule is surrounded by other atoms or molecules, the energy released via the electronic relaxation of the ionized species can be transferred to a neighbouring atom or molecule, which uses the released energy to emit one of its electrons. This process is called interatomic/intermolecular Coulombic decay $(\mathrm{ICD})^{1}$. Since the first prediction in 1997 (ref. 1), ICD has been extensively studied both theoretically and experimentally in a variety of weakly bound systems (for recent reviews, see refs 2,3 ). It is worth noting that ICD has also been observed in molecular water clusters, suggesting that ICD may also occur in waterabundant biological systems $s^{4,5}$ and be an important source of genotoxic low-energy electrons and radical cations.

Intriguingly it has been shown theoretically and verified experimentally that ICD is not an isolated phenomenon, but might be part of complex cascade relaxation mechanisms, occurring for instance following an Auger decay process ${ }^{6-14}$. To understand relaxation mechanisms, it is necessary to be aware of all possible channels and determine their significance, and in the present work, we investigated this subject.

In the regime of high-intensity radiation, Kuleff et al. ${ }^{15}$ proposed an ICD mechanism in which two neighbouring species are resonantly photo-excited to low-lying excited states, and the de-excitation of one of them leads to the ionization of the other via an ultrafast energy transfer. It was argued that this process could be an additional important ion-production mechanism in clusters exposed to intense resonant light, largely dominating the direct multi-photon ionization of clusters' constituents at moderate intensities. Recent experimental studies of large helium droplets excited to low-lying states further supported the high efficiency of this variant of the decay processes $^{16,17}$. What happens when the cluster constituents are brought to higher excited states?

In this article, we show that in this case different relaxation dynamics take place. We report the observation of a new type of ICD, as well as of a cascade of ICD processes. For ease of analysis and interpretation, we chose to study neon clusters. Ne clusters containing about 5,000 atoms were irradiated by free electron laser (FEL) pulses of $61 \mathrm{~nm}$ wavelength, corresponding to the $2 p^{6} \rightarrow 2 p^{5} 3 d$ atomic-like transition in the cluster ${ }^{18}$. The FEL pulse produces a multiply excited cluster which can relax either via ICD in which one of the excited $3 d$ electrons recombines with the $2 p$ hole, as shown in Fig. 1a, or with a much slower rate, by fluorescence. The present experiment, however, clearly demonstrated that this 'direct' ICD channel is significantly suppressed and instead, ICD transitions that lead to the final state $2 p^{5} 3 s$ state (see Fig. 1b) are dominant. Hereafter, we will refer to these transitions as 'intra-Rydberg' ICD. We have also observed secondary ICD processes (ICD cascades) resulting from the intermediate excited states such as $2 p^{5} 3 s$ populated by the primary ICD processes (see Fig. 1c). We note that similar processes have been studied in a completely different regime and form of matter, namely Rydberg gases, and shown to be responsible for the so-called avalanche ionization phenomenon a

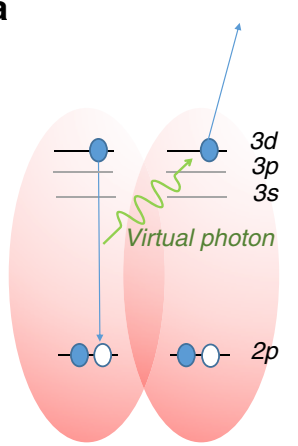

b

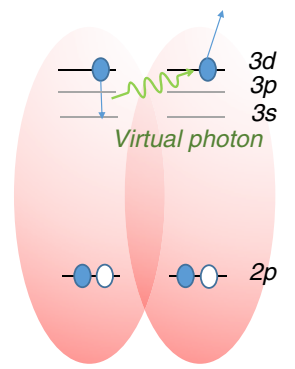

C

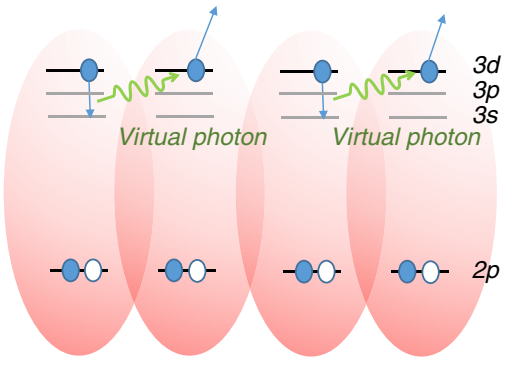

Ne cluster

Primary ICD : Intra-rydberg ICD
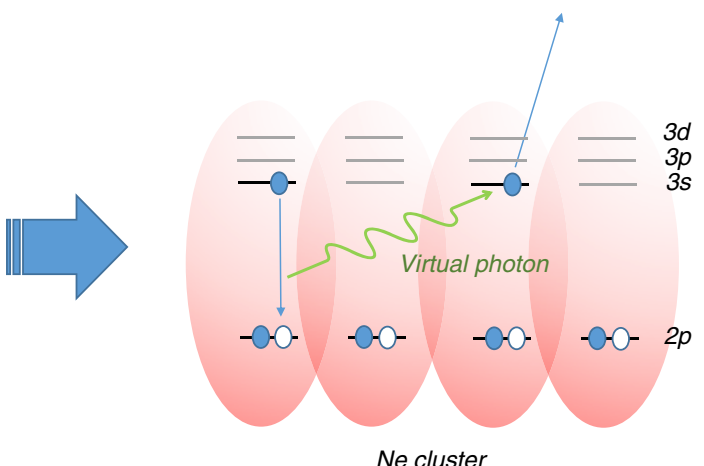

Secondary ICD

Figure 1 | Schematic diagram of Interatomic Coulombic Decay transitions from resonant excited states. (a) Schematic diagram of the direct Interatomic Coulombic Decay $(I C D)$ process: $\mathrm{Ne}^{\star}\left(2 p^{5} 3 d\right)+\mathrm{Ne}^{\star}\left(2 p^{5} 3 d\right) \rightarrow \mathrm{Ne}^{+}\left(2 p^{5}\right)+\mathrm{Ne}\left(2 p^{6}\right)+\mathrm{e}^{\mathrm{ICD}}$, which can be abbreviated as $3 d+3 d \rightarrow 2 p+\mathrm{e}^{\mathrm{ICD}}$. Here one excited neon atom, $\mathrm{Ne}^{\star}\left(2 p^{5} 3 d\right)$, returns to its ground state $\left(\mathrm{Ne}\left(2 p^{6}\right)\right)$ and the other neon atom is ionized by using the energy transferred by a virtual photon exchange. (b) Intra-Rydberg ICD: $3 d+3 d \rightarrow 3 s+e^{I C D}$. In this process, one $\mathrm{Ne}^{\star}\left(2 p^{5} 3 d\right)$ undergoes a transition to a lower excited state, $\mathrm{Ne}^{\star}\left(2 p^{5} 3 s\right)$, and the other one is ionized. Note that excited neon atoms remain in the neon cluster after intra-Rydberg ICD. (c) ICD cascade: after an intra-Rydberg ICD, the excited species thus produced continue to decay by secondary ICD processes either among themselves, or with other neighbouring excited species. Note that such an ICD cascade produces more ions compared with the case when the multiply-excited cluster would have decayed only by direct ICD processes. 
a

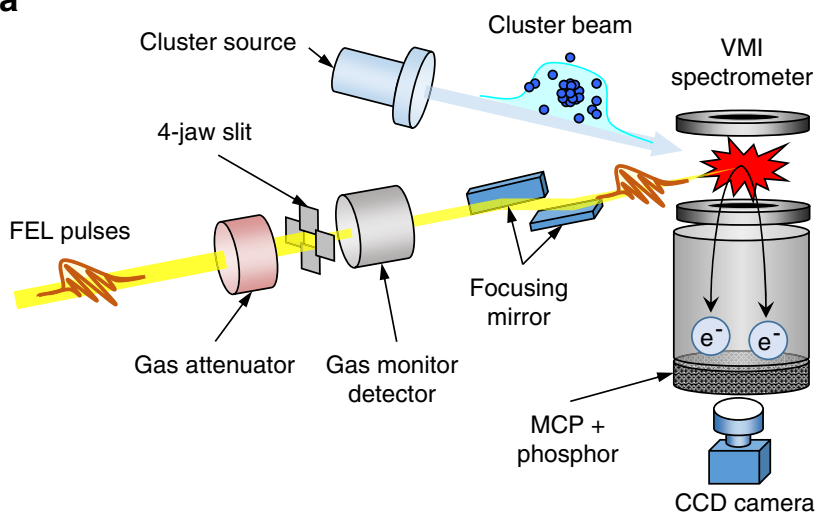

b

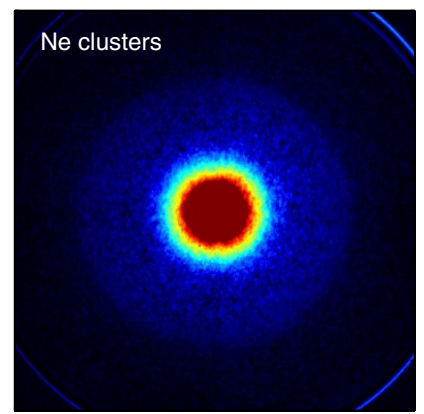

C

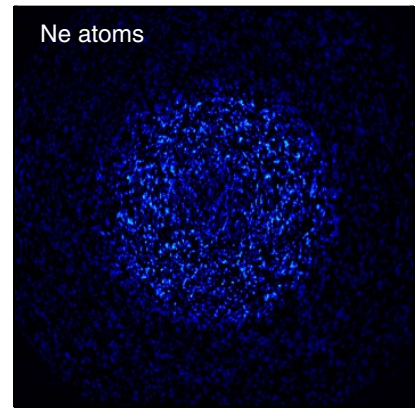

Figure 2 | Experimental set-up and raw experimental data. (a) Schematic drawing of experimental set-up (see Methods for details). (b) Velocity map images of electron distributions of Ne clusters. (c) Velocity map images of electron distributions of $\mathrm{Ne}$ atoms. The samples were exposed to $20.3 \mathrm{eV}$ Free Electron Laser FEL radiation at $\sim 4.5 \times 10^{11}$ and $\sim 5.3 \times 10^{12} \mathrm{~W} \mathrm{~cm}^{-2}$ (FEL) intensities, respectively. The VMI spectrometer voltages were set to detect electrons with kinetic energy up to $25 \mathrm{eV}$. Even at an order of magnitude lower intensity, we observe a much stronger electron signal in the case of clusters, showing the high efficiency of the Interatomic Coulombic Decay (ICD) as an electron-production process.

and the creation of ultracold plasma ${ }^{19-22}$. In Rydberg gases, however, collisions are recognized as a prerequisite for the ionizing mechanism, while in the van der Waals clusters studied by us the energy transfer between excited atoms is so efficient, due to the much smaller interatomic distances, that collisions (although not excluded) are not necessary for producing electrons and ions. Our study on clusters complements that on Rydberg gases and makes clear the importance of cascades in condensed matter.

\section{Results}

Experimental approach. The experiments were performed at the SPring-8 Compact SASE Source test accelerator in Japan ${ }^{23}$. Intense EUV-FEL pulses at $20.3 \mathrm{eV}$ photon energy (bandwidth of $0.3 \mathrm{eV}$ full-width at half-maximum (FWHM)) and of $30 \mathrm{fs}$ duration were focused on a $\mathrm{Ne}$ cluster beam. The kinetic energy distribution of the emitted electrons was measured with a velocity map imaging (VMI) spectrometer (see Fig. 2a, Methods and ref. 24). Electron velocity map images for Ne clusters and atoms are shown in Fig. 2. The photon energy of $20.3 \mathrm{eV}$ corresponds to the resonant excitation energy in a cluster of ground-state $\mathrm{Ne}$ atoms to the $2 p^{5} 3 d$ states. Despite the high FEL intensity, it is evident from Fig. 2 that the FEL pulse does not cause any significant electron emission from isolated atoms despite its high intensity $\left(\sim 5.3 \times 10^{12} \mathrm{~W} \mathrm{~cm}^{-2}\right)$. The situation changes dramatically for clusters, where a strong

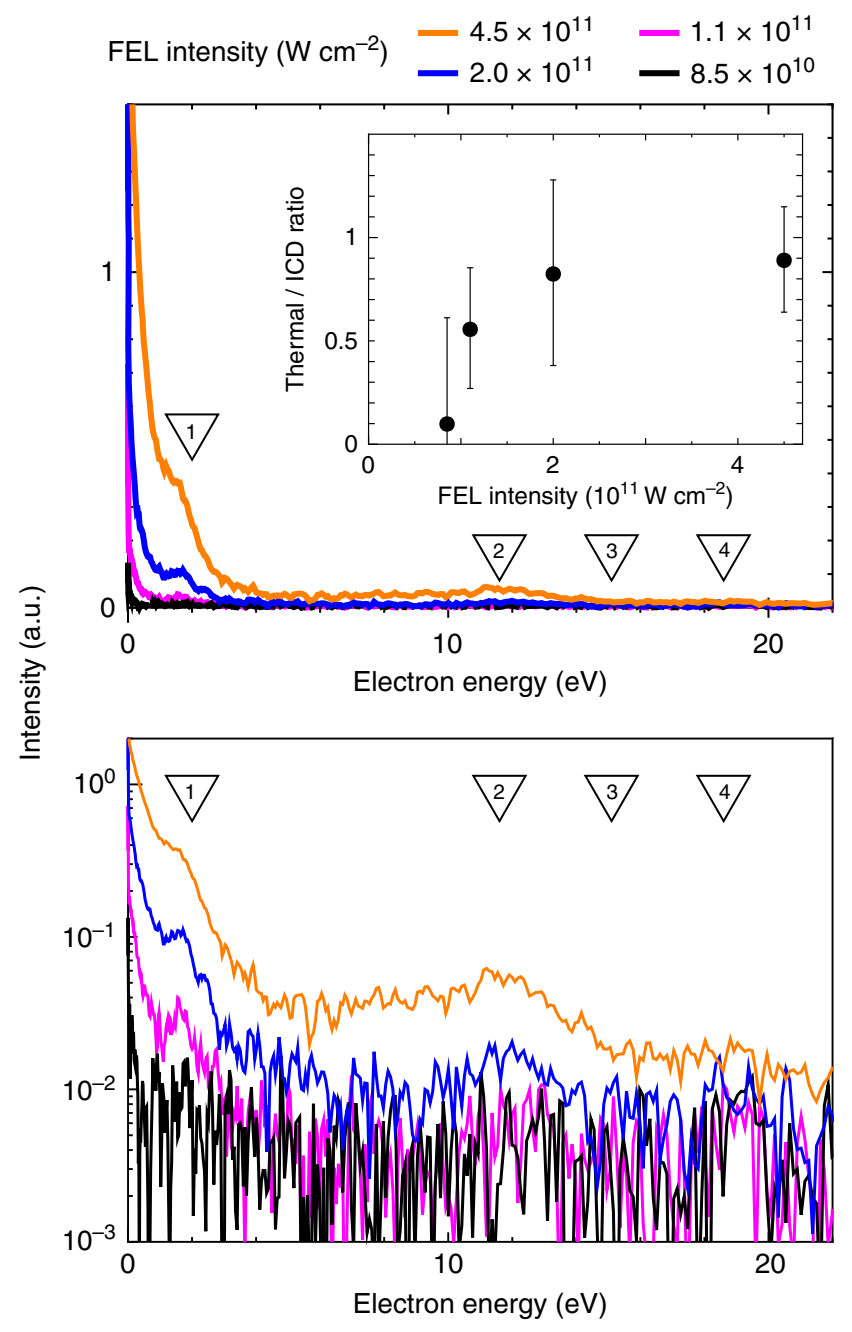

Figure 3 | Electron emission spectra of Ne clusters. Electron emission spectra of Ne clusters ( $\sim 5000)$ irradiated by $20.3 \mathrm{eV}$ photons at different Free Electron Laser (FEL) intensities. The $y$-axis scale of lower panel is logarithmic. Inset: The intensity ratio of thermal electrons to Interatomic Coulombic Decay (ICD) electrons is plotted as filled circles with error bars. Error bars represent the uncertainty in estimated intensity ratio. The triangles indicate the ICD electron energies calculated using the atomic energy levels, labelled as in Table 1.

electron signal is produced already at moderate FEL intensities $\left(\sim 4.5 \times 10^{11} \mathrm{~W} \mathrm{~cm}^{-2}\right)$ due to the ICD processes.

Electron emission spectra of neon clusters and assignment. Electron spectra from $\mathrm{Ne}$ clusters are shown in Fig. 3. On top of the well-known exponential thermal distribution of the electrons ${ }^{25}$, stemming from the increase of the cluster potential by charge accumulation and nanoplasma formation, we also observe distinct structures at about 1.8 and $11 \mathrm{eV}$ that become more pronounced with increasing FEL intensity.

Before assigning the observed structures, let us first consider the possible ICD transitions in Ne clusters under the present conditions, using the atomic energy level data for $\mathrm{Ne}$ (ref. 26). Table 1 summarizes all energetically allowed transitions. Apart from the $3 d$ to $2 p$ transition, the $3 d$ to $3 s$ transition in one $\mathrm{Ne}$ atom is able to eject a $3 d$ electron from a neighbouring excited atom. This intra-Rydberg ICD process can be viewed as a 'super Coster-Kronig' transition since all participating electrons occupy $n=3$ shells and, as we will see, dominates the other ICD 
Table 1 | Electron energies of possible transitions.

\begin{tabular}{|c|c|c|c|c|c|}
\hline Label & $\begin{array}{l}\text { Primary ICD Process } \\
\text { (Intra-Rydberg ICD) }\end{array}$ & $\begin{array}{l}\text { Secondary ICD Process } \\
\text { (Cascade ICD) }\end{array}$ & $\begin{array}{l}\text { Estimated ICD } \\
\text { electron energy }\end{array}$ & $\begin{array}{l}\text { Observed ICD } \\
\text { electron energy }\end{array}$ & $\begin{array}{l}\text { Estimated } \\
\text { ICD lifetime }\end{array}$ \\
\hline 1 & $3 d+3 d \rightarrow 2 p+\mathrm{e}^{I C D}$ & & $18.6 \mathrm{eV}$ & - & $87.8 \mathrm{~ns}$ \\
\hline 2 & $3 d+3 d \rightarrow 3 s+e^{I C D}$ & & $2 \mathrm{eV}$ & $1.8 \mathrm{eV}$ & $9.2 \mathrm{fs}$ \\
\hline 3 & & $3 s+3 s \rightarrow 2 p+e^{I C D}$ & $11.6 \mathrm{eV}$ & $11 \mathrm{eV}$ & $18.3 \mathrm{ps}$ \\
\hline 4 & & $3 s+3 d \rightarrow 2 p+e^{I C D}$ & $15.1 \mathrm{eV}$ & - & $0.5 \mathrm{~ns}$ \\
\hline
\end{tabular}

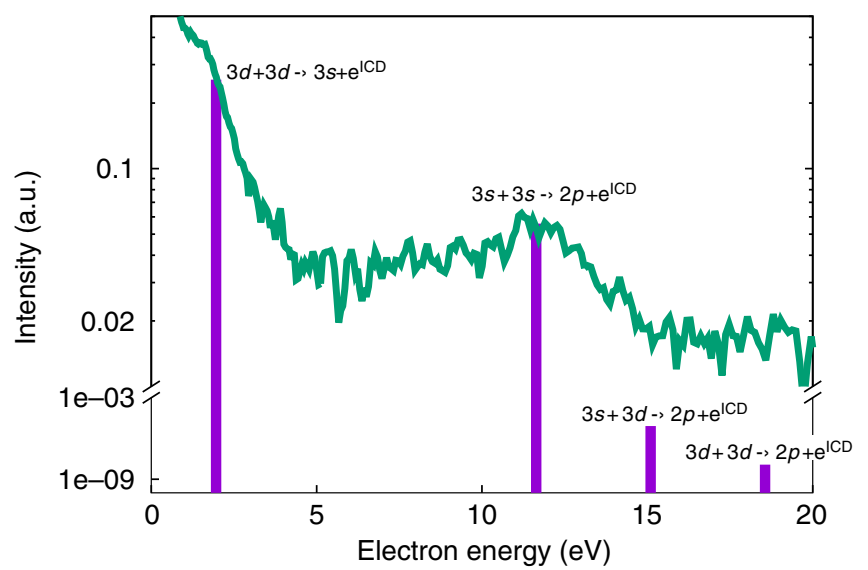

Figure 4 | Comparison between experimental data and theoretical simulations. Electron spectra (curves) and computations based on the solution of rate equations (vertical bars) given on a logarithmic scale. The electron spectra shown represent the experimental data recorded at $4.5 \times 10^{11} \mathrm{~W} \mathrm{~cm}^{-2}$. Note the break on the $y$-axis and the different scales below and above it. All Interatomic Coulombic Decay (ICD) transitions are also denoted. The computed data are normalized to the measured ones by the intensity of the peak at $\sim 12 \mathrm{eV}$. An exponential function accounting for the thermal distribution of the electron emission is extracted from the experimental spectra and added to the theoretical results, but not the experimental background.

processes. Moreover, the $\mathrm{Ne}^{\star}\left(2 p^{5} 3 s\right)$ produced by the intraRydberg ICD processes can continue to decay by ionizing neighbouring $\mathrm{Ne}^{\star}\left(2 p^{5} 3 d\right)$ or $\mathrm{Ne}^{\star}\left(2 p^{5} 3 s\right)$ (see Fig. 1c).

As we see from Table 1, an interacting pair of $\mathrm{Ne}^{\star}\left(2 p^{5} 3 d\right)$ atoms within a cluster can undergo 4 different ICD transitions (2 primary and 2 secondary) emitting electrons with different energies. These energies, obtained using atomic data, are shown with triangles in Fig. 3. The comparison of these ICD electron energies with the electron spectra suggests that the $1.8 \mathrm{eV}$ peak could be attributed to the $3 d+3 d \rightarrow 3 s+\mathrm{e}^{\mathrm{ICD}}$ decay route, while the structure around $11 \mathrm{eV}$ could stem from the secondary ICD process: $3 s+3 s \rightarrow 2 p+\mathrm{e}^{\mathrm{ICD}}$. The transition $3 s+3 d \rightarrow 2 p+\mathrm{e}^{\mathrm{ICD}}$ emitting electrons with $\sim 15 \mathrm{eV}$, and the 'direct' $3 d+3 d \rightarrow 2 p+$ $\mathrm{e}^{\mathrm{ICD}}$ transition $(\sim 18.6 \mathrm{eV})$ are not visible in the experimental spectra, as a consequence of their much lower decay rates (see below).

We note a small discrepancy between the estimated energies of the ICD electrons and the observed peak positions. One reason for the discrepancy could be the interatomic interaction potentials within the cluster that are not included in the estimation using atomic energy levels. Also, the energy of the emitted electrons may be shifted due to the increase of the cluster Coulomb field, as we discuss later.

Theoretical modelling of the ICD transition rates. To validate our interpretation of the measured electron spectra, we performed numerical simulations based on a system of rate equations. The system of equations describes the time evolution of the population of the excited atoms, which can decay pair-wise via an energetically allowed ICD transition with the corresponding rate. To obtain the rate of each possible ICD transition, we made use of the asymptotic formula for the ICD width $\Gamma$ (derived in ref. 15) which involves the oscillator strength $f$ of the de-excitation transition in one of the atoms in the pair, and the cross-section $\sigma$ to ionize the other excited electron in the other atom with the energy $\omega$ released by the de-excitation transition:

$$
\Gamma(R)=\frac{3 c}{\pi \omega^{2}} \frac{f \sigma}{R^{6}} .
$$

In the above expression, given in atomic units, $c$ denotes the speed of light and $R$ is the distance between the excited atoms. For all possible ICD transitions, the required atomic quantities (oscillator strengths and ionization cross-sections) were computed at the Hartree-Fock level.

The rate equations were solved for the number of electrons emitted in all possible ICD channels along the cascade (see Methods). The results are shown as a histogram in Fig. 4 together with the experimental data. The model cannot predict absolute intensities, but only relative ones, assuming all excited pairs have decayed and all emitted electrons have reached the detector with their initial energies. The model is also not sensitive to the initial concentration of $\mathrm{Ne}^{*}\left(2 p^{5} 3 d\right)$ and, therefore, to the FEL pulse intensity. That is why, to compare with the experimental spectrum, an exponential function accounting for the thermal distribution of the electron emission has been extracted from the experimental curve and added to the theoretical results, and the resulting data have been normalized to the intensity of the measured peak at $\sim 11.6 \mathrm{eV}$. We see that despite the simplicity of the model, it correctly describes the experimental observations: two strong peaks at $\sim 2$ and $\sim 11.6 \mathrm{eV}$ are predicted with an intensity ratio of approximately $2: 1$, while the transitions at $\sim 15.1$ and $\sim 18.6 \mathrm{eV}$ have very low rates and are thus not observed experimentally. The high-intensity of the line at $\sim 2 \mathrm{eV}$ is due to the high decay rate of the $3 d+3 d \rightarrow$ $3 s+\mathrm{e}^{\mathrm{ICD}}$ transition which nearly completely suppresses the 'direct' $3 d+3 d \rightarrow 2 p+\mathrm{e}^{\mathrm{ICD}}$ process (see column 6 in Table 1 ). According to our calculations, the $3 s+3 s \rightarrow 2 p+\mathrm{e}^{\mathrm{ICD}}$ transition is also much more efficient compared with the other secondary ICD transitions and, therefore, is the dominant decay mode in the second stage of the decay giving rise to the peak at $\sim 11.6 \mathrm{eV}$. We note that relaxing via a two-step ICD cascade produces up to 1.5 times more ions compared with the direct ICD transition, the upper limit corresponding to the case when all atoms that have relaxed to their $3 s$ excited state in the first ICD step decay pairwise in a second ICD transition.

\section{Discussion}

Let us discuss the intensity and the shape of the peak at around $11.6 \mathrm{eV}$. We see that the peak becomes more prominent at higher FEL intensities, has an asymmetric shape, and a developing 
plateau on the low-energy side. Bostedt et al. ${ }^{27}$ reported a similar feature in the photoelectron spectra of small argon clusters recorded at $38.7 \mathrm{eV}$. They suggested a multi-step ionization model, where sequential 1-photon-1-electron photoionization is assumed, and the plateau is attributed to the deceleration of the electrons by the developing attractive Coulomb potential of the cluster ion. In the present experiment, the $3 s+3 s \rightarrow 2 p+\mathrm{e}^{\mathrm{ICD}}$ process can take place after at least two primary ICD transitions have produced two positive charges in the cluster. Therefore, in the multi-step ICD process one can expect a deceleration of the ICD electrons by the accumulation of positive ions along the $3 d \rightarrow 3 s \rightarrow 2 p$ cascade, shifting the energies of the emitted ICD-electrons and thus forming a plateau in the electron spectrum. In addition, the increase of the cluster potential by charge accumulation would result in trapping the low-energy ICD electrons and thus nanoplasma formation. The intensity ratio of thermal electrons to ICD electrons increases with the increase in the FEL intensity up to $2.0 \times 10^{11} \mathrm{~W} \mathrm{~cm}^{-2}$ and becomes approximately constant in the FEL intensity range of $2.0 \times 10^{11}-4.5 \times 10^{11} \mathrm{~W} \mathrm{~cm}^{-2}$, as depicted in the inset of Fig. 3, suggesting that the source of thermal electrons could be trapping of the low-energy electrons created by the intra-Rydberg ICD by the charged cluster created by the ICD cascade.

Before concluding, a brief account will be given of the comparison between the present work and previously reported ones ${ }^{16,17,24}$. LaForge et al. ${ }^{16}$ and Ovcharenko et al. ${ }^{17}$ reported results on helium droplets at the resonance derived from the atomic $\mathrm{He} 1 s 2 p$ state. The energy of de-excitation from $1 s 2 p$ to $1 s^{2} s$ is, however, not sufficient to ionize another $1 s 2 p$ excited atom and thus is transferred only to the nuclear motion. In the present work, a novel phenomenon is observed for multiple excitation to higher Rydberg states, where the de-excitation energy of the intra-Rydberg transition can be transferred to another Rydberg atom and ionize it. Ovcharenko et al. clearly identified inelastic scattering of the energetic direct ICD electrons by the excited atoms and called it collective autoionization since more than two excited atoms are involved. In the present experiment, however, we could not identify such processes because of much lower density of excited atoms in the cluster, due to lower FEL intensity by more than one order of magnitude and lower inelastic scattering cross sections. The work of Yase et al. on the same excitation of neon clusters ${ }^{24}$ may be more closely related to the present work. However, that work focused on high power $\left(10^{13}-10^{14} \mathrm{~W} \mathrm{~cm}^{-2}\right)$ and observed different physics. There, the excited $3 d$ electrons were instantaneously delocalized and a nanoplasma was formed. As a result ICD was suppressed. In the present work, nanoplasma formation was significantly reduced by reducing FEL intensity and thus ICD could be observed.

In conclusion, in the electron emission spectra of Ne clusters exposed to intense EUV-FEL pulses producing multiple excitations to $3 d$ levels, we have identified a novel intra-Rydberg ICD mechanism occurring in the same electronic shell, which is much more efficient than the ICD to the ground state. Moreover, multiple population of higher Rydberg states in a cluster also triggers a very efficient cascade of ICDs in a step-wise de-excitation to the ground state, producing in that way more ions. A two-step cascade may produce up to $50 \%$ more ions compared with a single step relaxation. We note that, like all previously discovered ICD processes, these intra-Rydberg ICD and ICD cascades are expected to be general phenomena occurring in any weakly bound media (notably: water) exposed to intense light that is resonant with a higher-lying excited state of its constituents.

\section{Methods}

Experimental methods. The experiments were performed at the SPring- 8 Compact SASE Source (SCSS) test accelerator in Japan ${ }^{23}$. Intense EUV-FEL pulses were focused on a $\mathrm{Ne}$ cluster beam, and the kinetic energy distribution of emitted electrons was measured with a velocity map imaging (VMI) spectrometer ${ }^{28}$. The $\mathrm{Ne}$ clusters were prepared by adiabatic expansion of $\mathrm{Ne}$ gas through a $0.25 \mathrm{~mm}$ nozzle ${ }^{29}$. The Ne stagnation pressure was $10.8 \mathrm{bar}$, and the nozzle was cooled to $80 \mathrm{~K}$ using liquid nitrogen. The average cluster sizes $\langle\mathrm{N}\rangle$ were estimated to be about 5,000 atoms according to the well-known scaling law ${ }^{30,31}$. The FEL light source provided EUV pulses with a photon energy of $20.3 \mathrm{eV}$ (bandwidth was $0.3 \mathrm{eV}$ FWHM) and a duration of $30 \mathrm{fs}$ at a repetition rate of $30 \mathrm{~Hz}$. The FEL beam was steered by two upstream SiC plane mirrors, passed a gas attenuator, a 4 -jaw slit, and a gas monitor detector before entering the pre-focusing system of the beam line. The FEL intensity was reduced by the 4 -jaw slit down to $5 \%$ of full power and was adjusted by the gas attenuator. The skimmed FEL beam with a mean spot size of $14 \mu \mathrm{m}$ (FWHM) was focused onto the cluster beam at the center of the VMI spectrometer. The emitted electrons were accelerated toward a position-sensitive detector consisting of a set of micro channel plates (MCPs) followed by a phosphor screen, and recorded using a CCD camera synchronized to the arrival of the FEL pulse in the interaction chamber. The measured two-dimensional (2D) projection allows for the reconstruction of the three-dimensional (3D) momentum distribution of the ejected electrons using the pBASEX algorithm ${ }^{32}$. The energy scale of the electron spectrum was calibrated using the $3 \mathrm{p}$ photo-line of atomic argon $(I P=15.76 \mathrm{eV})$ with an energy resolution of $5 \%$.

Theoretical modelling. The present calculations were performed by solving a system of coupled rate equations that describes the population of the excited atoms decaying pair-wise by a cascade of ICD processes. For extracting the rate constant for each type of ICD transition, the Ne cluster was represented by a face-centered cubic system with a lattice constant of $4.4 \AA$ (corresponding to a nearest-neighbor distance of $3.1 \AA$ ) filling a sphere with a radius of $29.6 \AA$, resulting in a cluster of 5,017 neon atoms. Assuming that the FEL pulse can excite about $10 \%$ of the atoms in the cluster, the total decay rate (width) of each transition was obtained as the sum of the partial ICD widths, $\Gamma$, of all possible pairs of 500 randomly-distributed excited neon atoms estimated via equation (1). The latter was derived by expanding the Coulomb transition matrix element between the ICD initial and final electronic states of the dimer in a multipole series, and keeping only the dipole-dipole term $^{15,33}$. In this asymptotic approximation, the ICD width is the product of the transition probabilities for the radiative de-excitation in one of the atoms and for the photoionization of the other one. Those transitions are characterized by the excitation oscillator strength $f$ and by the photoionization cross-section $\sigma$ which were computed at the Hartree-Fock level. We note that although within this approximation the radiative transition $3 d \rightarrow 3 s$ is dipole-forbidden, the ICD process $3 d+3 d \rightarrow 3 s+\mathrm{e}^{\mathrm{ICD}}$ is allowed, since the outgoing electron carries the angular momentum required for the conservation of the total momentum in the Coulomb matrix element. Moreover, in a large cluster the orbital momentum is no longer a good quantum number as the atomic orbitals overlap locally to form mixed states. Thus, a strict distinction between $p, d$, and so on. Rydberg state is nearly impossible. The important difference is between intra- and inter-shell transitions. That is why, in order to estimate the ICD width for $3 d+3 d \rightarrow 3 s+\mathrm{e}^{\mathrm{ICD}}$ transition on the same level of theory, the oscillator strength of the atomic dipole-allowed $3 d \rightarrow 3 p$ transition was utilized in equation (1). This is a rough assumption, but the model is not very sensitive to the exact value of the oscillator strength. It can be a few orders of magnitude smaller and will still quench the $3 d+3 d \rightarrow 2 p+\mathrm{e}^{\mathrm{ICD}}$ process. This is a result of the other quantities entering in equation (1), the ionization cross-section $\sigma$ and the virtual photon energy $\omega$, which also largely favour the $3 d+3 d \rightarrow 3 s+\mathrm{e}^{\mathrm{ICD}}$ transition. The ionization cross-section for removing a $3 d$ electron with a $2 \mathrm{eV}$ photon (corresponding to the $3 d \rightarrow 3 \mathrm{~s}$ transition) is about 3 orders of magnitude larger than the cross-section for removing the same electron with a $19 \mathrm{eV}$ photon (corresponding to the $3 d \rightarrow 2 p$ transition). In addition, the decay rate depends as $1 / \omega^{2}$ on the virtual-photon energy.

We note also that since the model does not take the cluster dynamics into account and assumes that all excited pairs have decayed by ICD to produce electrons with the respective energies, it is not sensitive to the initial population of $\mathrm{Ne}^{\star}\left(2 p^{5} 3 d\right)$ in the cluster and, therefore, to the FEL intensity.

Data availability. The data that support the findings of this study are available from the corresponding author on reasonable request.

\section{References}

1. Cederbaum, L. S., Zobeley, J. \& Tarantelli, F. Giant intermolecular decay and fragmentation of clusters. Phys. Rev. Lett. 79, 4778-4781 (1997).

2. Averbukh, V. et al. Interatomic electronic decay processes in singly and multiply ionized clusters. J. Elec. Spectr. Rel. Phenom. 183, 36-47 (2011).

3. Jahnke, T. Interatomic and intermolecular Coulombic decay: the coming of age story. J. Phys. B 48, 082001 (2015).

4. Jahnke, T. et al. Ultrafast energy transfer between water molecules. Nat. Phys. 6, 139-142 (2010).

5. Mucke, M. et al. A hitherto unrecognized source of low-energy electrons in water. Nat. Phys. 6, 143-146 (2010). 
6. Santra, R. \& Cederbaum, L. S. Coulombic energy transfer and triple ionization in clusters. Phys. Rev. Lett. 90, 153401 (2003).

7. Morishita, Y. et al. Experimental evidence of interatomic coulombic decay from the Auger final states in argon dimers. Phys. Rev. Lett. 96, 243402 (2006).

8. Kreidi, K. et al. Localization of inner-shell photoelectron emission and interatomic Coulombic decay in $\mathrm{Ne}_{2}$. J. Phys. B: At. Mol. Opt. Phys. 41, 101002 (2008).

9. Yamazaki, M. et al. Decay channel dependence of the photoelectron angular distributions in core-level ionization of Ne dimers. Phys. Rev. Lett. 101, 043004 (2008).

10. Gokhberg, K., Kolorenc, P., Kuleff, A. I. \& Cederbaum, L. S. Site- and energyselective slow-electron production through intermolecular Coulombic decay. Nature 505, 661-663 (2014).

11. Kimura, M. et al. Efficient site-specific low-energy electron production via interatomic Coulombic decay following resonant Auger decay in argon dimers. Phys. Rev. A 87, 043414 (2013).

12. O'Keeffe, P. et al. The role of the partner atom and resonant excitation energy in interatomic coulombic decay in rare gas dimers. J. Phys. Chem. Lett. 4, 1797-1801 (2013).

13. Kimura, K. et al. Controlling low-energy electron emission via resonant-Augerinduced interatomic Coulombic decay. J. Phys. Chem. Lett. 4, 1838-1842 (2013).

14. Trinter, F. et al. Resonant Auger decay driving intermolecular Coulombic decay in molecular dimers. Nature 505, 664-666 (2014).

15. Kuleff, A. I., Gokhberg, K., Kopelke, S. \& Cederbaum, L. S. Ultrafast interatomic electronic decay in multiply excited clusters. Phys. Rev. Lett. 105, 043004 (2010).

16. Ovcharenko, Y. et al. Novel collective autoionization process observed in electron spectra of He clusters. Phys. Rev. Lett. 112, 073401 (2014).

17. LaForge, A. C. et al. Collective autoionization in multiply-excited systems: a novel ionization process observed in helium nanodroplets. Sci. Rep. 4, 3621 (2014).

18. Kloiber, T., Laasch, W., Zimmerer, G., Coletti, F. \& Debever, J. M. Observation of total and partial photon-stimulated desorption yields of neutral atoms from solid $\mathrm{Ne}$ under selective excitation in the excitonic range. Europhys. Lett. 7, 77 (1988).

19. Robert-de-Saint-Vincent, M. et al. Spontaneous avalanche ionization of a strongly blockaded Rydberg Gas. Phys. Rev. Lett. 110, 045004 (2013).

20. Tanner, P. J., Han, Jianing, Shuman, E. S. \& Gallagher, T. F. Many-body ionization in a frozen Rydberg gas. Phys. Rev. Lett. 100, 043002 (2008).

21. Amthor, T., Reetz-Lamour, M., Westermann, S., Denskat, J. \& Weidemüller, M. Mechanical effect of van der Waals interactions observed in real time in an ultracold Rydberg gas. Phys. Rev. Lett. 98, 023004 (2007).

22. Hung, J., Sadeghi, H., Schulz-Weiling, M. \& Grant, E. R. Evolution from Rydberg gas to ultracold plasma in a supersonic atomic beam of Xe. J. Phys. B: At. Mol. Opt. Phys. 47, 155301 (2014).

23. Shintake, T. et al. A compact free-electron laser for generating coherent radiation in the extreme ultraviolet region. Nat. Photon. 2, 555-559 (2008).

24. Yase, S. et al. Crossover in the photoionization processes of neon clusters with increasing EUV free-electron-laser intensity. Phys. Rev. A 88, 043203 (2013).

25. Arbeiter, M. \& Fennel, T. Ionization heating in rare-gas clusters under intense XUV laser pulses. Phys. Rev. A 82, 013201 (2010).

26. Kramida, A., Ralchenko, Yu., Reader, J. \& NIST ASD Team. NIST Atomic Spectra Database (ver. 5.2) http://physics.nist.gov/asd (2015).

27. Bostedt, C. et al. Multistep ionization of argon clusters in intense femtosecond extreme ultraviolet pulses. Phys. Rev. Lett. 100, 133401 (2008)

28. Gryzlova, E. V. et al. Doubly resonant three-photon double ionization of Ar atoms induced by an EUV free-electron laser. Phys. Rev. A 84, 063405 (2011).
29. Sugishima, A. et al. Charge and energy transfer in argon-core-neon-shell clusters irradiated by free-electron-laser pulses at $62 \mathrm{~nm}$. Phys. Rev. A 86 033203 (2012).

30. Hagena, O. \& Obert, W. Cluster formation in expanding supersonic jets: effect of pressure, temperature, nozzle size, and test gas. J. Chem. Phys. 56, 1793-1802 (1972).

31. Karnbach, R., Joppien, M., Stapelfeldt, J., Wörner, J. \& Möller, T. CLULU: An experimental setup for luminescence measurements on van der Waals clusters with synchrotron radiation. Rev. Sci. Instrum. 64, 2838-2849 (1993).

32. Garcia, G. A., Nahon, L. \& Powis, I. Two-dimensional charged particle image inversion using a polar basis function expansion. Rev. Sci. Instrum. 75, 4989-4996 (2004).

33. Averbukh, V., Müller, I. B. \& Cederbaum, L. S. Mechanism of interatomic Coulombic decay in clusters. Phys. Rev. Lett. 93, 263002 (2004).

\section{Acknowledgements}

We are grateful to late Dr Mitsuru Nagasono for an invaluable contribution and the SPring-8 Compact SASE Source Test Accelerator Operation Group at RIKEN for continuous support in the course of the studies. This work was supported by the X-ray Free Electron Laser Utilization Research Project and the X-ray Free Electron Laser Priority Strategy Program of the Ministry of Education, Culture, Sports, Science and Technology of Japan (MEXT), by the Grant-in-Aid for the Global COE Program 'The Next Generation of Physics, Spun from Universality and Emergence' from the MEXT, by the Grants-in-Aid $(20310055,21244062)$ from the Japan Society for the Promotion of Science (JSPS), by the Proposal Program of SACLA Experimental Instruments of RIKEN and by the IMRAM project. N.V.G. thanks IMPRS-QD for financial support. L.S.C. and A.I.K. acknowledge the financial support of ERC under AdG no 227597 and partially of DFG Research Unit 1789. P.V.D. acknowledges financial support from the LOEWE focus-project ELCH.

\section{Author contributions}

The experiment was conceived by K.N., A.I.K., M.Yao and K.U. The experiment was prepared and carried out by K.N., K.Ma, H.F., K.Mo, T.N., T.S., T.T., S.M., S.W., K.C.P., C.C., C.M., N.S., M.Yab and K.U. Theoretical calculations were performed by N.V.G., P.V.D., L.S.C. and A.I.K. Detailed data analysis was performed by K.N., D.I. and K.Ma. The manuscript was drafted by K.N., D.I., A.I.K. and K.U. in consultation with all authors.

\section{Additional information}

Competing financial interests: The authors declare no competing financial interests.

Reprints and permission information is available online at http://npg.nature.com/ reprintsandpermissions/

How to cite this article: Nagaya, K. et al. Interatomic Coulombic decay cascades in multiply excited neon clusters. Nat. Commun. 7, 13477 doi: 10.1038/ncomms13477 (2016).

Publisher's note: Springer Nature remains neutral with regard to jurisdictional claims in published maps and institutional affiliations.

(c) (i) This work is licensed under a Creative Commons Attribution 4.0 International License. The images or other third party material in this article are included in the article's Creative Commons license, unless indicated otherwise in the credit line; if the material is not included under the Creative Commons license, users will need to obtain permission from the license holder to reproduce the material. To view a copy of this license, visit http://creativecommons.org/licenses/by/4.0/

(C) The Author(s) 2016 